\title{
Telemetria na transmissão de dados de desempenho de máquinas agrícolas utilizando tecnologias GSM/GPRS e ZigBee
}

\author{
Telemetry in transmission of performance data of agricultural machines using GSM/GPRS and ZigBee
}

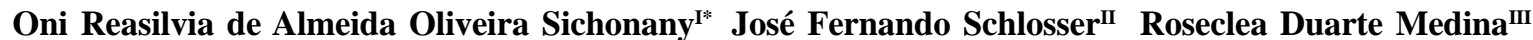 \\ Iria Brucker Roggia ${ }^{\mathrm{I}}$ Jaziel Souza Lôbo ${ }^{\mathrm{IV}}$ Fernado Beux dos Santos $^{\mathrm{IV}}$
}

\begin{abstract}
- NOTA-
\section{RESUMO}

Este trabalho apresenta os testes de um sistema de telemetria (T-SADA) que foi adaptado ao gerenciamento da mecanização agrícola utilizando uma colhedora automotriz autopropelida na colheita de soja e arroz irrigado. O sistema consiste na coleta e envio de dados de um ponto móvel (a máquina agrícola) para uma base fixa (um computador servidor) e faz a integração de dois meios de transmissão e a validação dos dados (propiciando tolerância a falhas). Os meios de transmissão de dados usados foram a tecnologia da telefonia móvel, GSM/GPRS, e rádio-frequência, através do

two means of transmission and validation of data (providing fault tolerance). The means of data transmission used were the technology of mobile telephony, GSM / GPRS and radio frequency through the Zigbee. The T-SADA is redundant because it has more than one form of transmission, which ensures the transmission of information even if a media is not able to operate correctly at any given time. Priority is given to the transmission GSM / GPRS and leaving the ZigBee as an alternative means, controlled by an engine fault detection. The results were satisfactory ensuring the transmission of data with fault tolerance and redundantly in remote locations and in different relief.
\end{abstract} Zigbee. O T-SADA é redundante, pois possui mais de uma forma de transmissão, o que garante o envio das informações mesmo se um dos meios não tiver condições de operar corretamente num determinado momento. É dada prioridade para a transmissão GSM/GPRS e deixando o ZigBee como meio alternativo, controlado por um mecanismo de detecção de falhas. Os resultados alcançados foram satisfatórios, garantindo a transmissão dos dados com tolerância a falhas $e$ de forma redundante em locais remotos e em diferentes relevos.

Palavras-chave: aquisição de dados, telefonia móvel, rádiofrequência.

\section{ABSTRACT}

This paper presents the tests of a telemetry system (T-SADA) to be adapted to the management of agricultural mechanization using a self-propelled combine harvester to harvest soybeans and rice. The system consists of collecting and sending data of a moving point (a farm machine) to a fixed base (a computer server) and makes the integration of
Key words: data acquisition, mobile telephony, radio frequency.

O gerenciamento da frota de máquinas tem se tornado cada vez mais importante no planejamento, execução e controle das operações agrícolas por estar diretamente relacionado com a capacidade de combinar terra, trabalho e capital para a obtenção de um retorno com lucro satisfatório (SCHLOSSER, 2003). Os custos da maquinaria em relação aos custos totais da exploração de uma propriedade rural podem alcançar percentuais elevados, chegando até quarenta por cento. O estudo das operações agrícolas, levando-se em conta a capacidade de trabalho e a eficiência de campo, visa a racionalizar o emprego das máquinas, implementos e ferramentas na execução dos trabalhos

\footnotetext{
IPrograma de Pós-graduação em Engenharia Agrícola, Universidade Federal de Santa Maria (UFSM), Rua Jorge Pedro Abelin, 158/ 03, 97050-390, Santa Maria, RS, Brasil. E-mail: oni@inf.ufsm.br. *Autor para correspondência.

"Departamento de Engenharia Rural, UFSM, Santa Maria, RS, Brasil.

IIIDeparmento de Eletrônica e Computação, UFSM, Santa Maria, RS, Brasil.

IvPrograma de Pós-graduação em Informática, UFSM, Santa Maria, RS, Brasil.
} 
(VIEIRA \& BRIZOLLA, 2009). Assim, é conveniente determinar o comportamento das máquinas em operação, observando de maneira mais rigorosa detalhes como consumo de combustível, velocidade da operação e largura de trabalho da máquina.

A utilização de redes de sensores em uma máquina agrícola, conectados a um sistema de tomada de dados, permite alcançar os objetivos acima, possibilitando medir uma série de parâmetros como os citados. Todos esses dados coletados remotamente necessitam ser transmitidos para uma central de armazenamento para o seu monitoramento, avaliação e controle. A telemetria é a transferência e utilização de dados provindos de uma ou mais máquinas remotas, permitindo uma comunicação instantânea, via rede de computadores fixa ou sem fio (wireless) (BONDE, 2009). Entre os elementos que compõem um sistema de telemetria estão os sensores, que monitoram, controlam e medem algum tipo de atividade.

Nas redes sem fio, o espaço livre serve como meio de transmissão de dados para o envio de informações, tomando-se como exemplo a comunicação por rádio-frequência ou por estrutura de telefonia móvel. O protocolo ZigBee IEEE 802.15.4 é um padrão de rede usado em redes de dispositivos sem fio que requerem baixo consumo de energia, baixo custo e alta confiabilidade, baseado em uma norma aberta global, recomendada para transmissão de dados de sensores (ANDRIGHETTO, 2008).

A rede de telefonia móvel, também conhecida como tecnologia Global System for Mobile communications (GSM), promove toda infraestrutura para ofertar o serviço de transmissão de dados com maior ou menor taxa de transmissão nos mais diversos lugares, inclusive os de difícil acesso. Para isso, utiliza-se de operadoras de telefonia celular que mantêm a concessão de serviços em todo o mundo (CARAM, 2008). O protocolo General Packet Radio Service (GPRS) é uma tecnologia de dados disponível em redes GSM, cuja taxa de transmissão de dados típica é de 26 a $40 \mathrm{kbits} \mathrm{s}^{-1}$. A maior parte do território considerado agrícola, no Brasil, se mantém com tecnologias GSM/GPRS.

O Sistema de Apoio à Decisão para utilização no Agronegócio (SADA) é um protótipo de aplicação criado para auxiliar gerentes do agronegócio nas tomadas de decisões relativas ao controle e ao bom desempenho operacional das máquinas agrícolas. Seu intuito é monitorar o desempenho da máquina em trabalho e alertar as operações errôneas realizadas, buscando melhorar a eficiência e reduzir os custos operacionais. O usuário (gerente), utilizando dispositivos portáteis como telefones celulares, smartphones e/ou notebooks, pode ter acesso a uma infraestrutura de serviços, mantendo-a durante o seu deslocamento, com a operação agrícola sendo executada ou não .

O presente trabalho faz parte do projeto SADA, consistindo no sistema responsável pela transmissão dos dados obtidos da máquina agrícola para um computador servidor. Este sistema de telemetria, chamado T-SADA, possui tratamento de falhas e redundância de transmissão através do uso de dois meios de transmissão, que são a estrutura de telefonia móvel (GSM/GPRS) e rádio-frequência (protocolo ZigBee). Após a coleta, os dados são transferidos, sem a utilização de cabos ou fios, para um computador servidor de forma on-line e autônoma (sem a interferência do operador), definindo um sistema de comunicação entre a base de dados e a máquina agrícola, que se encontra em movimento constante.

A rede do T-SADA é constituída de dois pontos, um móvel e um fixo. O Ponto de Acesso Móvel (PAM), instalado na máquina agrícola, é composto de sensores com as funções de medida de consumo de combustível (fluxômetro), altura da plataforma de corte (potenciômetro analógico) e definição de posicionamento geográfico e velocidade, por meio de um GPS. Também faz parte do PAM, um datalogger modelo CR 1000 da marca Campbell, que tem como finalidade a aquisição dos dados junto aos sensores e a comunicação com os módulos transmissores GSM/ GPRS e Xtend/Zigbee. O Ponto de Acesso Fixo (PAF) refere-se a uma infraestrutura mínima com uma antena instalada em uma estrutura metálica a 25,0 metros do solo, localizada em um ponto estratégico, conforme geografia do território analisado. Também faz parte, um dos rádios Xtend/Zigbee conectado à antena e a um notebook que possui uma conexão com a Internet.

Para executar o sistema de transmissão, foram implementados softwares tanto no PAM quanto no PAF, que possibilitam o gerenciamento da redundância e da tolerância a falhas. O termo redundância no escopo deste trabalho consiste na repetição ou duplicação, cuja função é garantir a execução e a comunicação, apesar da existência de possíveis falhas de transmissão. A redundância do TSADA é obtida pelo emprego de duas tecnologias de transmissão, ou seja, duas redes que funcionam independentemente uma da outra, que são estrutura de telefonia celular e rádio-frequência, utilizadas de forma a garantir o envio dos dados. Se um meio perde a conexão, o outro passa a transmitir. Já a tolerância a falhas é a técnica que permite manter a integridade e a segurança dos dados do sistema, que continuará a executar com dados corretos mesmo na presença de falhas.

No PAM, o sistema consiste em fazer a leitura dos sensores em ciclos definidos no tempo de 
quatro segundos, gravando os dados gerados em memória permanente em forma de tabelas dinâmicas. Na redundância, a transmissão é feita somente por um dos meios de cada vez para diminuir o processamento e, consequentemente, o consumo de energia. $\mathrm{O}$ meio utilizado como prioritário é o GSM, por apresentar uma maior taxa de transmissão de dados e porque o sistema Xtend/ZigBee tem vantagem em possuir um rápido sincronismo na sua conexão, no caso de falhar o sistema prioritário. O tempo de sincronismo no GSM varia de 10 a 30 segundos, enquanto que no sistema Xtend/ ZigBee leva em média 30 milesegundos. O controle de redundância ainda gerencia a retomada da transmissão prioritária, voltando imediatamente a transmitir por GSM e deixando novamente o sistema Xtend em espera.

No PAF, o sistema é encarregado de receber e armazenar os dados, fazendo a validação das informações para garantir a integridade e armazenar com segurança, ou então descartar os pacotes falhos. A inconsistência dos pacotes é determinada de duas formas: a) pela falta de informações de identificação e/ ou por problema nos dados coletados pelos sensores e b) pela quebra de sequência nos registros das coletas feita no PAM, estabelecidas através de um número. Para os pacotes inconsistentes, o PAF faz um pedido de reenvio ao PAM, que os retransmite. O pacote a ser transmitido consiste em uma string, que contém caracteres identificadores de início e fim, \# e @, respectivamente, e uma definição de duas partes, a primeira destinada a um cabeçalho e a segunda aos dados coletados. No cabeçalho constam informações sobre a integridade do pacote, como a definição do tamanho completo da string e a identificação se é uma mensagem original ou um reenvio, pedido pelo PAF. Se houver falhas na análise dos caracteres de início e fim e no cabeçalho da mensagem, o pacote é descartado, havendo o pedido de reenvio.

Os testes foram realizados em uma área agrícola pertencente ao município de São Francisco de Assis, no estado do Rio Grande do Sul, Brasil, em diferentes locais dentro da propriedade rural, utilizando uma colhedora automotriz autopropelida marca Massey Ferguson, modelo 5650, em seis dias de trabalho diferentes e em duas culturas, soja e arroz irrigado. Em cada cultura, foram feitos testes com duas operadoras de telefonia celular, para a transmissão da tecnologia GSM/GPRS. A diferença entre as culturas do arroz e da soja no ponto de vista de transmissão sem fio é o relevo do terreno, que, no caso do arroz, é uma planície sem apresentar obstáculos entre as antenas de transmissão (visada direta) e, na soja, o terreno é ondulado apresentando alguns obstáculos.
Durante os dois primeiros dias e os dois últimos, foram avaliadas as atividades na cultura do arroz, em que, no primeiro dia e nos dois últimos, não foi realizada nenhuma transmissão com o sistema GPRS, em função da baixa presença de sinal GSM na localidade, enquanto que, no segundo dia, alguns dados foram transmitidos por essa tecnologia. De 2.749 registros recebidos, apenas 256 foram por GSM/GPRS, correspondendo a $9,31 \%$ do total.

No terceiro e quarto dias de avaliação, utilizando a cultura da soja, o comportamento se apresentou de forma similar, no terceiro dia de coleta $39,17 \%$ dos registros transmitidos foram recebidos pelo sistema de telefonia móvel e no quarto dia não houve presença de recepção por esta tecnologia, fato semelhante nos demais dias testados.

Analisando os pedidos de reenvio, podese averiguar que, quase em sua totalidade, os registros pedidos foram recebidos, com exceção de três solicitações via Xtend/ZigBee, que se perderam e não foram transmitidos, representando 0,19\% do total. Este valor é considerado insignificante, levando em conta o número de registros total que foi de 13.133, não resultando em prejuízo para a análise das informações.

Os testes demonstraram que a redundância foi eficaz, garantindo a transmissão com a troca de tecnologia sem degradação do tempo, já que, em caso de desconexão do modem GSM, o sistema leva 30 milisegundos para conectar ao Xtend e enviar os dados.

O sistema de reenvio consegue determinar a transmissão de dados falhos em tempo hábil, de até 5 segundos na maioria dos casos, sendo que o ciclo de coleta é de 4 segundos. O reenvio, portanto, possibilitou uma análise dos dados de forma on-line, com um tempo considerado pequeno entre o ciclo e o reenvio, permitindo assim um retorno imediato, com resultados obtidos pelo processamento dos dados.

Apesar do sinal GSM/GPRS ser precário no local do experimento, o Xtend/ZigBee estabeleceu a cobertura da maioria da área experimental avaliada. Mesmo em casos em que não houve a cobertura dos sistemas, foi possível recuperar os dados não transmitidos através do processo de reenvio no momento de re-conexão.

O sistema T-SADA alcançou os objetivos propostos, ou seja, garantiu a transmissão dos dados com tolerância a falhas e de forma redundante em locais remotos e de relevos diferenciados, tais como planície e com declividade.

\section{REFERÊNCIAS}

ANDRIGHETTO, E. Sistema de processamento de sinais biomédicos: rede wireless zigbee com aplicação do padrão 
IEEE 802.15.4. 2008. 163f. Dissertação (Mestrado em Engenharia Elétrica) - Universidade Federal de Santa Catarina, Florianópolis, SC.

BONDE, I. (s.d.). Telemetria. Disponível em: $<$ www.teleco.com.br/tutoriais/tutorialmtelemetria/ pagina_2.asp>. Acesso em: 03 fev. 2009.

CARAM, V.O. Gerenciamento do espectro de frequências, otimização e compartilhamento de rede móvel em múltiplas tecnologias. 2008. 142f. Dissertação (Mestrado em Engenharia Elétrica) - Universidade de Brasília, Brasília, DF.

SCHLOSSER, J.F. Módulo 6: administração de máquinas agrícolas. Santa Maria: UFSM/NEMA, 2003. 100p. (Série Técnica).

VIEIRA, E.P.; BRIZOLLA, M.M. A influência da mecanização da atividade agrícola na composição do custo de produção. Disponível em: <http://www.furb.br/congressocont/ _files/CCG\%20152.pdf>. Acesso em: 15 abr. 2009. 